\title{
To Develop a Security System for Vehicles Using Arduino UNO
}

\author{
Jagdish Pimple ${ }^{1}$, Mahesh Warghane ${ }^{2}$, Shashank Shivrekar ${ }^{3}$, Mayur Meshram ${ }^{4}$, Shashank \\ Bhimte $^{5}$ \\ ${ }^{1}$ Head and Assistant professor, ${ }^{2,3,4,5}$ UG Student \\ Department of Information Technology, Nagpur Institute of Technology, Nagpur, India,440026
}

\begin{abstract}
In the modern era as we are developing new vehicles with great technologies, concern about our vehicles safety is also increasing. And we are living in the age of vehicle thefts, so vehicle safety has become our matter of prime importance. As a solution to the given mentioned problem, we have developed a prototype model of a fingerprint based security system for vehicles by interfacing Fingerprint sensor model R306 along with Arduino Uno. An user of a vehicle can start it but only upon it's fingerprint authentication.
\end{abstract}

Keywords- Arduino Uno, Fingerprint sensor R306, Android Application.

\section{I- INTRODUCTION}

S ecurity regarding vehicles is the growing concern in India. Protection of vehicles against theft is one of the major issue of concern. For enhancing the security of vehicles against various thefts Embedded Computing is widely used. In recent times many techniques have been tried and tested to protect and secure the automobile. In previous years Radiofrequency Identification (RFID) cards were designed for ignition start of automobile [5]. However, the chances of losing the card or get stolen can led to the failure of the system.

Dr. Saritha et al. proposed a Fingerprint based security system for vehicles using Arduino Uno and Fingerprint sensor module R307 [4]. In there system, a person can start the vehicle on successful authentication of fingerprint which eventually put that vehicle into motion. There they have allowed the user to enter maximum 5 attempts for authenticating the fingerprint. To add and store a fingerprint they have used 3 buttons there. These buttons were facilitating the adding and removing of the fingerprint. They have also used a LCD for displaying all of their modules functionality such as fingerprint inclusion and deletion. But the problem with this system is that, if there occurs the failure of the LCD panel then the system is of no use i.e. if the LCD panel face some hardware problem then the user will be unable to see what exactly they have to do for authentication.

Interfacing Arduino with the Fingerprint sensor enables us to store multiple number of fingerprints [1,9]. Since bio-metrics is the reliable security technology, we can use the fingerprints for authentication of the users identification. Also it is an accurate digital identification solution [7]. Also there are various security systems which works on GPS and GSM modules [2,6]. All these System includes the high data speed internet for security concerns.

Nandakumar et al. proposed a system for real time vehicle security through face recognition [8]. They have used the LDA algorithm for the face recognition. Also they have used various relays and IR sensors for their system. This was a completely image processing based module.

\section{II- PROPOSED WORK}

In this paper, we propose a method which uses a fingerprint control for ignition of the vehicle. In previous system there are some potholes. As various mechanical arrangements can be done to manipulate the system. But in our proposed system we are connecting our system's hardware with the software application so whenever the thief wants to start the vehicle, system will trigger the message to the user immediately. 
For wide range of connectivity we have used a GSM module in our system. GSM provides global connectivity for its users. We've used the GSM module SIM900A.

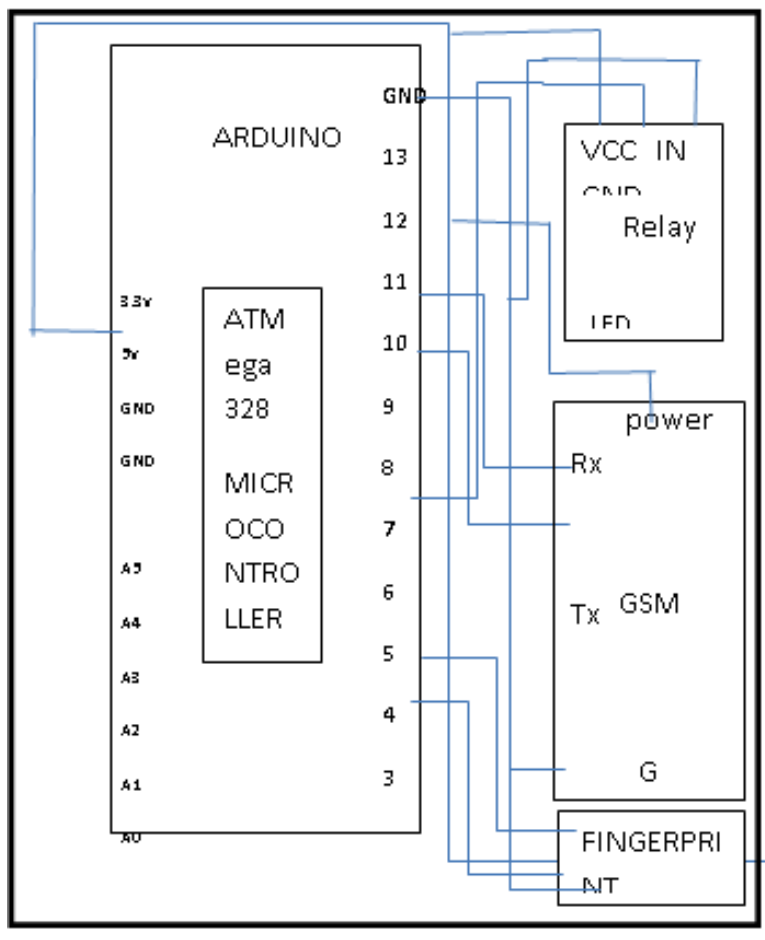

Fig- Block Diagram of proposed system

For authentication of the fingerprints we've used the Fingerprint Module R306 sensor. The fingerprint sensor module has two sub-modules: fingerprint Enrolment and Fingerprint Recognition. The finger print enrolment module adds and stores the fingerprints of all the users who are authorized to drive the vehicle.

Fingerprint matching sub-module compares the input fingerprint against one or more templates that are present in the database. The user is allowed n number of attempts for authentication. The fingerprint matching techniques are minutiae-based matching and pattern matching type.

For starting a vehicle a user requires to give his/her fingerprint onto the sensor. This fingerprint is matched against one or more fingerprints in the database. The microcontroller ATmega328 of the Arduino Uno board will receive the signals sent by the Fingerprint sensor module R306. The signals sent by the Fingerprint module are nothing but the fingerprints which are given by the user. ATmega328 microcontroller handles user authentication. It first matches the scanned fingerprint with those fingerprints are stored in its database. And after matching if it is true then it eventually starts the vehicle. This all is done by the microcontroller ATmega328.

Now, when an unauthorized user tries to start the vehicle, his/her fingerprints mismatches with the fingerprints that are stored in the database, and with the help of the GSM module it sends the message to the user with whom which that particular vehicle is registered.

We've used an Android application for the user to monitor hi/her vehicle when someone tries to harm the kit of ignition system or forcefully tries to start the vehicle. Also, if the user wants to give his/her vehicle to someone else then the user also has the capability to start the system by his/her android application. For this purpose we've used some buttons in our application. Below given figure represents the block diagram of the system which comprises of the connectivity with the sensors, GSM module and power Supply. The hardware used here operates on $+5 \mathrm{~V}$ and $+3.3 \mathrm{~V}$ supply.

\section{III- HARDWARE COMPONENTS}

\section{Arduino Uno}

Arduino Uno is a circuit board which has an Arduino Ide. These two together gives the user to embed various codes for achieving their goal. This Arduino Ide uses the embedded C language. Together they are capable of reading analog or digital input signals from different sensors, activating the motor, turning LED on/off and do many other such activities. All these functionalities are performed by sending a set of instructions to the ATtmega328 main microcontroller, on the board via Arduino IDE. For power supply the Arduino is provided with a USB cable. Also it has a Power (Barrel Jack), voltage regulator, crystal oscillator, voltage pins $(3.3 \mathrm{v}, 5 \mathrm{v}$, gnd, vin), A0 to A5 analog pins, icsp pin, power led indicator, tx and rx leds, 14 digital input/output pins, Aref, and Arduino reset.

\section{Fingerprint Module R306}


Here in order to validate the authenticate user we have used the Fingerprint sensor R306 module. It consists of an optical fingerprint sensor and highspeed DSP processor. Also this module consists of the high performance fingerprint alignment algorithm this algorithm helps the module for the simple entry of the fingerprint and its matching. This hardware consists of the high-capacity FLASH chips, hardware and software composition, stable performance, image processing and many other functions.

\section{GSM Module SIM900A}

The SIM900A is a complete Dual-band GSM/GPRS solution in as SMT module which can be embedded in the customer applications. This is an ultra compact and reliable wireless module. Featuring an industry-standard interface, the SIM900A delivers GSM/GPRS 900/1800MHz performance for voice, SMS, Data, and Fax in a small form factor and with a tiny configuration of $24 \mathrm{~mm} * 24 \mathrm{~mm} * 3 \mathrm{~mm}$. This module can fit into almost all the space requirements in user applications, especially for slim and compact demand of design.

\section{Relay}

A relay is an electrically operated switch. Many relays use an electromagnet to mechanically operate a switch, but other operating principles are also used, such as solid-state relays. Relays are used where it is necessary to control a circuit by a low-power signal for this it requires a complete isolation between control and controlled circuits. Due to this capability of the relay we have used it in our work. It has 3 pins, first is for the VCC i.e the power supply, IN for interfacing with the Arduino Uno and a GND pin. It works on the principle of an electromagnetic attraction.

\section{IV-SOFTWARE COMPONENT}

We've made an Android application for interaction with the user. Instead of this android application we should've used the LCD module but due to its sensitivity we have not used that module. To give various advantages to the user we have made this application. When user wants to give his/her vehicle to some other person he/she just have to operate the vehicle by this application itself. This application is easy to install. This application has several buttons which are made to trigger some events regarding the ignition of the vehicle.

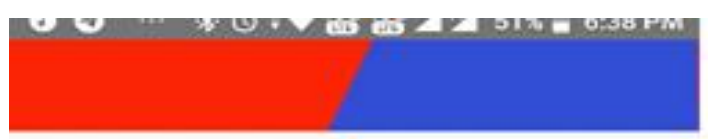

NIT BIKE ATHENTICATOR

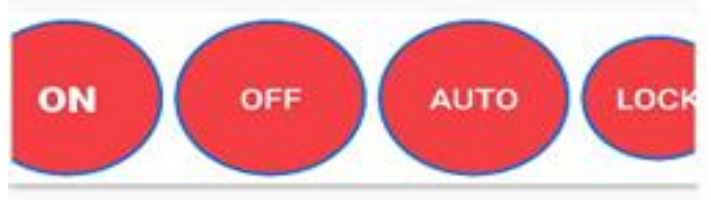

The above given screenshot is of the application that we've developed. It includes several buttons, as we can see in the above image.

\section{V- CONCLUSION}

Our work is aimed at enhancing and improving the security standards of the vehicles. We have developed an embedded system that uses the Fingerprint biometric authentication for igniting a vehicle. Also with this hardware we also have made an Android application which is interfaced with this hardware, and together they make an interactive system for vehicle security. Vehicles having security systems like this will help in reducing theft of a vehicle.

\section{REFERENCES}

[1] Khin San Myint, Chan Mya Mya Nyein "Fingerprint Based Attendance System Using Arduino" 2018 International Journal of Scientific and Research Publications, Volume 8, Issue 7.

[2] Mohammad A. Al-Kheder and Sharaf A. Al-Kheder, "Intelligent Anti-Theft and Tracking System for Automobiles", International Journal of ML and computing, 2012.

[3] N. Kiruthiga, L. latha, S. Thangasamy, "Real Time Biometric based Vehicle Security System with GPS and GSM Technology", Procedia Computer Science 47471 479, 2015. 
[4] Dr. Saritha Nambodiri, Arun P. "Fingerprint based security system for vehicles", Int. Journal of Advance Research, Ideas And Innovations In Technology.

[5] Upendran Rajendran and Albert Joe Francis, "Anti Theft Control System Design Using Embedded System”, Proc. IEEE, Vol. 85, Page no. 239-242, 2011.

[6] B. Rama Murthy, O. Jagadish, K. Tanveer Alam, V. Mahammad Dada, K. Priyanka Gandhi, "Development of GSM Based Advanced Alert Home Locker Safety Security System Using Arduino UNO”, 2018 IJSRST, Volume 4, Issue 2.

[7] Shubham Sharma, Rahul Sharma, "Combining Keystroke Security and Finger Print Identification for Mobile Based Secure Authentication System", International Journal of Computer Science and Mobile Applications, Vol.6, Issue 2, page 21-30, Feb-2018.

[8] C. Nandakumar, G. Muralidharan and N. Tharami, "Real Time Vehicle Security System through Face Recognition", International Review of Applied Engineering Research, vol.4, pp.371-378,Nov-2014.

[9] A. Karthikeyan "FINGERPRINT BASED IGNITION SYSTEM" International Journal of Computational Engineering Research, vol.2, pg 236-43, 2012. 